\title{
Trimethylcyanosilane as a Convenient Reagent for the Preparation of Trimethylsilyl Enol Ethers of 1,3-Diketones
}

\author{
Jin-Cong Zhuo \\ College of Pharmacy, The Ohio State University, Columbus, Ohio 43221, USA \\ Tel.: (614)-688-3149, Fax: (614)-292-2435, E-mail: zhuo.1@ osu.edu
}

Received: 22 August 1999 / Accepted: 12 September 1999 / Published: 10 October 1999

\begin{abstract}
Trimethylsilyl enol ethers of 1,3-diketones are generated "in situ" or obtained in high isolated yield by the reaction of 1,3-diketones with trimethylcyanosilane in various solvents such as cyclohexane, hexane, benzene, methylene chloride, chloroform, carbon tetrachloride, and acetonitrile.
\end{abstract}

Keywords: Trimethylcyanosilane, Silylation, 1,3-Diketones, Trimethylsilyl enol ethers.

\section{Introduction}

Silyl enol ethers of 1,3-diketones are effective silylating agents and useful precursors for the preparation of Diels-Alder dienes, 1,3-bis(silyloxy)buta-1,3-dienes. They have found extensive application in organic synthesis. The preparation of the silyl enol ethers of 1,3-diketones resulted in only fair yields by using reagents such as trimethylchlorosilane/strong bases [1], bis(trimethylsilyl)formamide [2], bis(trimethylsilyl)acetamide [3], triethylsilyl-thiobenzene [4], trimethylsilyl trifluoromethanesulfonate [5], and ethyl trimethylsilylacetate/tetra-n-butylamonium fluoride [6], employed previously for the silylation of carbonyl compounds. A few methods for the preparation of trimethylsilyl enol ethers of 1,3-diketones have been developed by using hexamethyldisilazane [7], hexamethyldisilazane/imidazole [8], 2-oxo-3-trimethylsilyltetrahydro-1,3-oxazole [9], and (trimethylsilyl)diethylamine /methyl iodide [10].

(C) 1999 by the authors. Reproduction of this article, by any means, is permitted for noncommercial purposes. 
Trimethylcyanosilane $\left(\mathrm{Me}_{3} \mathrm{SiCN}\right)$ has been used widely in organic synthesis $[11,12]$. It has been used to protect a variety of alcohols, phenols, carboxylic acids, amines and thiols [13]. Previously we have shown that $\alpha$-cyano enols were conveniently converted to trimethylsilyl enol ethers by using $\mathrm{Me}_{3} \mathrm{SiCN}$ [14]. We now wish to report a method for the silylation of 1,3-diketones to their trimethylsilyl enol ethers by using $\mathrm{Me}_{3} \mathrm{SiCN}$.

\section{Results and Discussion}

The reaction of $\mathrm{Me}_{3} \mathrm{SiCN}$ with 1,3-diketones is complicated as shown in Scheme 1. The products formed depend upon the experimental conditions: temperature, time, the stoichiometric amount of $\mathrm{Me}_{3} \mathrm{SiCN}$ added, and the catalyst used [11]. The reaction of pentan-1,3-dione (1) with excess $\mathrm{Me}_{3} \mathrm{SiCN}$ formed trimethylsilyl enol ether $\mathbf{2}$ at room temperature or the adduct $\mathbf{3}$ at elevated temperatures $\left(90^{\circ} \mathrm{C}\right)$ $[11,15]$. Alternatively, the reaction yielded compounds 4 or/and 5 under different conditions, [15-17].

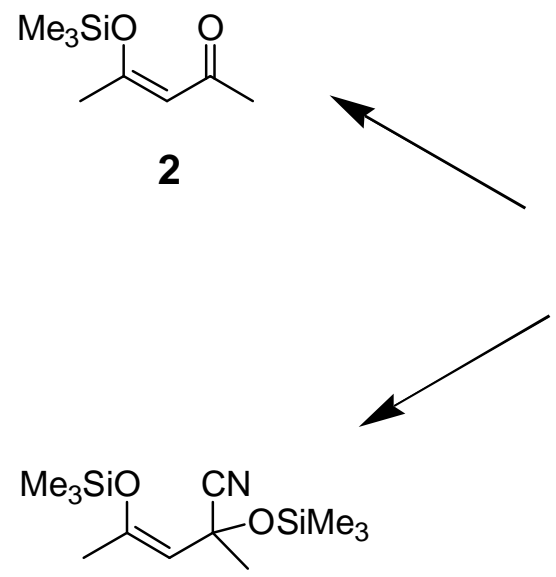

3<smiles>CC=C(C(C)=O)C(C)=O</smiles>

1

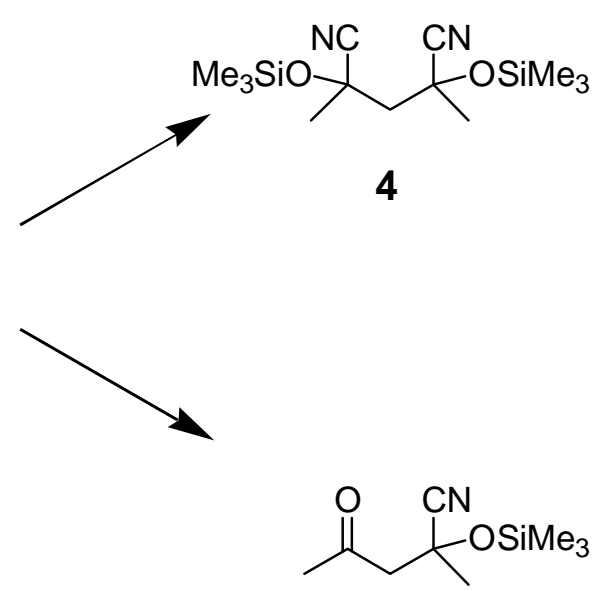

5

\section{Scheme 1.}

We observed that the silylation proceeded very smoothly at ambient temperature when $\mathrm{Me}_{3} \mathrm{SiCN}$ was added to the 1,3-diketones $\mathbf{6 a - f}$ or $\mathbf{8 a -} \mathbf{- g}$ in $\mathrm{CDCl}_{3}$ solution. The reactions were completed in 10 min (monitored by ${ }^{1} \mathrm{H}$ NMR) and produced the corresponding trimethylsilyl enol ethers $7 \mathbf{a}-\mathbf{f}$ or $\mathbf{9 a - g}$ in quantitative yield (Scheme 2). The reactions of $\mathrm{Me}_{3} \mathrm{SiCN}$ with 1,3-diketones can be carried out in various solvents. For example, the reaction of $\mathrm{Me}_{3} \mathrm{SiCN}$ with cyclohexane-1,3-dione $\mathbf{6 b}$ proceeded very smoothly in cyclohexane, hexane, benzene, carbon tetrachloride, chloroform, methylene chloride, and acetonitrile, and afforded $\mathbf{7 b}$ in quantitative yield. 


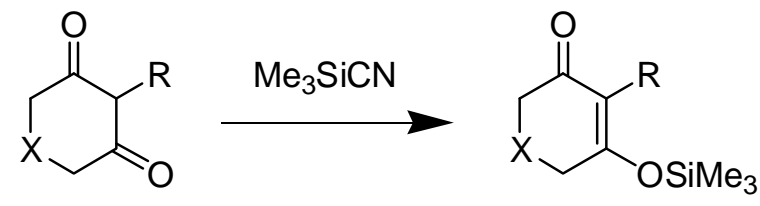

6a-f

7a-f

\begin{tabular}{|l|l|l||l|l|l|}
\hline & $\mathrm{X}$ & $\mathrm{R}$ & & $\mathrm{X}$ & $\mathrm{R}$ \\
\hline $\mathbf{a}$ & - & $\mathrm{H}$ & d & - & $\mathrm{Me}$ \\
b & $\mathrm{CH}_{2}$ & $\mathrm{H}$ & e & $\mathrm{CH}_{2}$ & $\mathrm{Me}$ \\
c & $\mathrm{CMe}_{2}$ & $\mathrm{H}$ & $\mathbf{f}$ & $\mathrm{CMe}_{2}$ & $\mathrm{Me}$ \\
\hline
\end{tabular}

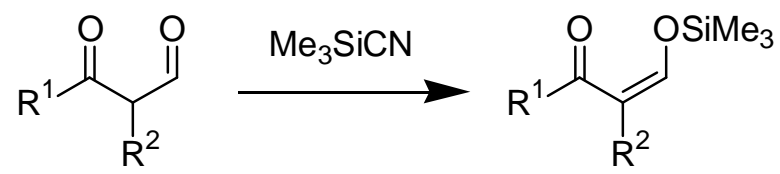

8a-g

9a-g

\begin{tabular}{|l|ll||l|ll|}
\hline & $\mathrm{R}^{1}$ & $\mathrm{R}^{2}$ & & $\mathrm{R}^{1}$ & $\mathrm{R}^{2}$ \\
\hline a & $\mathrm{Me}$ & $\mathrm{H}$ & $\mathbf{e}$ & $\mathrm{Ph}$ & $\mathrm{Me}$ \\
$\mathbf{b}$ & $\mathrm{Ph}$ & $\mathrm{H}$ & $\mathbf{f}$ & $\mathrm{Ph}$ & $\mathrm{Ph}$ \\
c & $\mathrm{Me}$ & $\mathrm{Me}$ & $\mathbf{g}$ & \multicolumn{2}{|c}{$\left(\mathrm{CH}_{2}\right)_{4^{-}}$} \\
d & $\mathrm{Et}$ & $\mathrm{Me}$ & & & \\
\hline
\end{tabular}

Scheme 2.

The reaction of $\mathrm{Me}_{3} \mathrm{SiCN}$ with 2-acetylcyclopentanone 10a gave a ca. 1:1 ratio mixture of the two trimethylsilyl enol ethers 11a and 12a in quantitative yield (Scheme 3). Interestingly, the reaction of $\mathrm{Me}_{3} \mathrm{SiCN}$ toward 2-acetylcyclohexanone $\mathbf{1 0 b}$ was slow and produced only trimethylsilyl enol ether 12b in $50 \%$ yield after $24 \mathrm{~h}$.

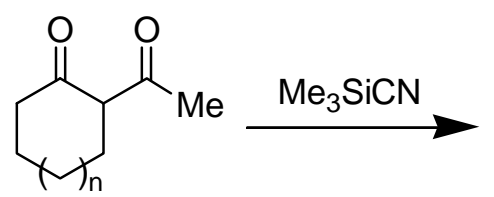<smiles>COC(C)=C1CCCCC1=O</smiles>

11a $\mathrm{n}=0$

$10 \mathbf{a} \mathrm{n}=0$
$\mathbf{1 0 b} \mathrm{n}=1$

$11 \mathrm{~b} n=1$<smiles>CO[Si](C)(O)C1=C(C(C)=O)CCCC1</smiles>

12a $\mathrm{n}=0$

12b $\mathrm{n}=1$

Scheme 3. 


\section{Conclusion}

In summary, we have shown that $\mathrm{Me}_{3} \mathrm{SiCN}$ is an efficient silylation reagent for silylating 1,3diketones, especially for the cyclic 1,3-diketones and 2-(hydroxymethylene)ketones. The method provides a convenient way to trimethylsilyl enol ethers of 1,3-diketones under neutral conditions.

\section{Experimental}

The general procedure for the preparation of trimethylsilyl enol ethers of 1,3-diketones is as follows: $\mathrm{Me}_{3} \mathrm{SiCN}(0.205 \mathrm{~g}, 2.05 \mathrm{mmol})$ was added to 1,3-diketone $(2 \mathrm{mmol})$ in dry $\mathrm{CDCl}_{3}(3 \mathrm{ml})$ or other suitable solvent under a nitrogen atmosphere. The progress of the reaction was directly monitored by ${ }^{1} \mathrm{H}-\mathrm{NMR}$. The reaction is generally completed at room temperature in 5-10 min. After the completion of the reaction, a stream of nitrogen was allowed to pass through the mixture for $5 \mathrm{~min}$. The resulting solution contains the trimethylsilyl enol ether. The solvent was evaporated under reduced pressure. The residue is the desired compound.

3-(Trimehylsilyloxy)cyclopent-2-en-1-one (7a): ${ }^{1} \mathrm{H} \mathrm{NMR}\left(\mathrm{CDCl}_{3}\right): 5.30$ (t, J = 1.0, H-2), 2.57 (m, 2H, H-4), 2.42 (m, 2H, H-5), 0.33 (s, 9H, $\mathrm{OSiMe}_{3}$ ).

3-(Trimehylsilyloxy)cyclohex-2-en-1-one (7b): ${ }^{1} \mathrm{H} \mathrm{NMR}\left(\mathrm{CDCl}_{3}\right): 5.37$ (t, J = 0.7, H-2), 2.36 (td, $\mathrm{J}=$ 6.2, 0.7, 2H, H-4), 2.32 (t, J = 6.7, 2H, H-6), 1.97 (m, 2H, H-5), 0.30 (s, 9H, OSiMe $)_{3}$.

5,5-Dimethyl-3-(trimehylsilyloxy)cyclohex-2-en-1-one (7c): ${ }^{1} \mathrm{H} \mathrm{NMR}\left(\mathrm{CDCl}_{3}\right): 5.35$ (t, J = 0.6, H2), 2.24 (d, J = 0.6, 2H, H-4), 2.17 (s, 2H, H-6), 1.07 (s, 6H, $2 \mathrm{Me}$ ), 0.30 (s, 9H, OSiMe $)_{3}$.

2-Methyl-3-(trimehylsilyloxy)cyclopent-2-en-1-one (7d): ${ }^{1} \mathrm{H} \mathrm{NMR}\left(\mathrm{CDCl}_{3}\right): 2.51$ (m, 2H, H-4), 2.43 (m, 2H, H-5), 1.58 (t, J = 1.8, Me), 0.33 (s, 9H, $\mathrm{OSiMe}_{3}$ ).

2-Methyl-3-(trimehylsilyloxy)cyclohex-2-en-1-one (7e): ${ }^{1} \mathrm{H} \mathrm{NMR}\left(\mathrm{CDCl}_{3}\right): 2.40$ (tq, $\mathrm{J}=6.4,1.6$, 2H, H-4), 2.35 (t, J = 6.7, 2H, H-6), 1.94 (m, 2H, H-5), 1.66 (t, J = 1.6, Me), 0.28 (s, 9H, OSiMe $)_{3}$.

5,5-Dimethyl-2-methyl-3-(trimehylsilyloxy)cyclohex-2-en-1-one (7f): ${ }^{1} \mathrm{H} \mathrm{NMR}\left(\mathrm{CDCl}_{3}\right): 2.26$ (q, $\mathrm{J}=$ 1.5, 2H, H-4), 2.23 (s, 2H, H-6), 1.67 (t, J = 1.5, 3H, Me), 1.07 (s, 6H, $2 \mathrm{Me}$ ), 0.28 (s, 9H, OSiMe $)$.

4-(Trimehylsilyloxy)but-3-en-2-one (9a). ${ }^{1} \mathrm{H} \mathrm{NMR}\left(\mathrm{CDCl}_{3}\right): 7.51(\mathrm{~d}, \mathrm{~J}=12.2,1 \mathrm{H}, \mathrm{H}-4), 5.73(\mathrm{~d}, \mathrm{~J}=$ 12.2, 1H, H-3), 2.17 (s, 3H, Me), 0.29 (s, 9H, $\mathrm{OSiMe}_{3}$ ).

1-Phenyl-3-(trimehylsilyloxy)prop-2-en-1-one (9b). ${ }^{1} \mathrm{H} \mathrm{NMR}\left(\mathrm{CDCl}_{3}\right): 7.87-7.91$ (m, 2H, $\left.\mathrm{Ph}\right), 7.75$ (d, J = 11.6, 1H, H-3), 7.50-7.55 (m, 1H, Ph), 7.42-7.47 (m, 2H, Ph), 6.53 (d, J = 11.6, 1H, H-2), 0.32 (s, 9H, $\mathrm{OSiMe}_{3}$ ).

3-Methyl-4-(trimehylsilyloxy)but-3-en-2-one (9c). ${ }^{1} \mathrm{H} \mathrm{NMR}\left(\mathrm{CDCl}_{3}\right): 7.46$ (q, J = 1.2, 1H, H-4), 2.17 (s, 3H, MeCO), 1.70 (d, J = 1.2, Me-3), 0.30 (s, 9H, OSiMe).

2-Methyl-1-(trimehylsilyloxy)pent-1-en-3-one (9d). ${ }^{1} \mathrm{H} \mathrm{NMR}\left(\mathrm{CDCl}_{3}\right): 7.48$ (q, J = 1.3, 1H, H-4), 2.55 (q, J = 7.4, 2H, H-4), 1.72 (d, J = 1.3, Me-2), 1.10 (t, J = 7.4, 3H, H-5), 0.29 (s, 9H, OSiMe $)_{3}$.

2-Methyl-1-phenyl-3-(trimehylsilyloxy)prop-2-en-1-one (9e). ${ }^{1} \mathrm{H} \mathrm{NMR}\left(\mathrm{CDCl}_{3}\right): 7.53$ (m, 2H, $\mathrm{Ph}$ ), $7.48(\mathrm{~m}, 1 \mathrm{H}, \mathrm{Ph}), 7.41$ (m, 2H, Ph), 7.15 (q, J = 1.3, 1H, H-3), 1.88 (d, J = 1.3, 3H, Me-2), 0.22 (s, 9H, 
$\left.\mathrm{OSiMe}_{3}\right)$.

1,2-Diphenyl-3-(trimehylsilyloxy)prop-2-en-1-one (9f). ${ }^{1} \mathrm{H} \mathrm{NMR}\left(\mathrm{CDCl}_{3}\right)$ : 7.63-7.67 (m, 2H), 7.21$7.49(\mathrm{~m}, 9 \mathrm{H}), 0.24\left(\mathrm{~s}, 9 \mathrm{H}, \mathrm{OSiMe}_{3}\right)$.

2-(Trimehylsilyloxymethylene)cyclohexan-1-one $(\mathbf{9 g}) .{ }^{1} \mathrm{H} \mathrm{NMR}\left(\mathrm{CDCl}_{3}\right): 7.46(\mathrm{t}, \mathrm{J}=2.0,1 \mathrm{H},=\mathrm{CH})$, 2.44 (td, J = 6.7, 2.0, 2H, H-3), 2.34 (t, J = 6.7, 2H, H-6), 1.77-1.90 (m, 2H, H-5), 1.64-1.76 (m, 2H, $\mathrm{H}-4), 0.26$ (s, 9H, $\mathrm{OSiMe}_{3}$ ).

2-(1-Trimehylsilyloxyethylene)cyclopentan-1-one (11a). ${ }^{1} \mathrm{H} \mathrm{NMR}\left(\mathrm{CDCl}_{3}\right): 2.59$ (tq, $\mathrm{J}=7.3,1.8$, $2 \mathrm{H}, \mathrm{H}-3), 2.48-2.52(\mathrm{~m}, 2 \mathrm{H}, \mathrm{H}-5), 2.32(\mathrm{t}, \mathrm{J}=1.8,3 \mathrm{H}, \mathrm{Me}), 1.76-1.87$ (m, 2H, H-4), $0.26(\mathrm{~s}, 9 \mathrm{H}$, $\left.\mathrm{OSiMe}_{3}\right)$.

2-(1-Oxoethyl)-1-(Trimehylsilyloxy)cyclopent-1-ene (12a). ${ }^{1} \mathrm{H} \mathrm{NMR}\left(\mathrm{CDCl}_{3}\right):$ 2.47-2.52 (m, 4H, H3 and $\mathrm{H}-5$ ), 2.34 (s, 3H, MeCO), 1.76-1.87 (m, 2H, H-4), 0.32 (s, 9H, OSiMe ${ }_{3}$ ).

2-(1-Oxoethyl)-1-(Trimehylsilyloxy)cyclohex-1-ene (12b). ${ }^{1} \mathrm{H} \mathrm{NMR}\left(\mathrm{CDCl}_{3}\right): 2.37$ (s, 3H, $\mathrm{MeCO}$ ), 2.30-2.36 (m, 2H), 2.20-2.28 (m, 2H), 1.64-1.71 (m, 2H), 1.51-1.58 (m, 2H), 0.29 (s, 9H, OSiMe $)_{3}$.

Acknoledgement: The author is indebted to Professor Hugo Wyler for valuable comments.

\section{References and Notes}

1. (a) West, R. J. Am. Chem. Soc. 1958, 80, 3246-3249; (b) House, H. O.; Czuba, L. J.; Gall, M. Olmstead, H. D. J. Org. Chem. 1969, 34, 2324-2336; (c) Pawlenko, S. in Houben-Weyl, Methoden der Organischen Chemie. 4th Edn. In: Muller, E.; Bayer, O. Editors. Vol. XIII/5, Georg Thieme Verlag, Stuttgart, 1980, 193-201.

2. Kantlehner, W.; Kugel, W.; Bredereck, H. Chem. Ber. 1972, 105, 2264-2270.

3. (a) Dedier, J.; Gerval, P.; Frainnet, E. J. J. Organomet. Chem. 1980, 185, 183-197; (b) Klebe, J. F.; Finkbeiner, H.; White, D. M. J. Am. Chem. Soc. 1966, 88, 3390-3395.

4. Ojima, I.; Nagai, Y. J. Organomet. Chem. 1973, 57, C42-C44.

5. (a) Emde, H.; Domsch, D.; Feger, H.; Frick, U.; Goetz, A.; Hergott, H. H.; Hofmann, K.; Kober, W.; Kraegeloh, K.; Oesterle, T.; Steppan, W.; West, W.; Schimchen, G. Synthesis 1982, 1-26; (b) Emde, H.; Goetz, A.; Hofmann, K.; Simchen, G. Justus Liebigs Ann. Chem. 1981, 1643-1657; (c) Simchen, G.; Kober, W. Synthesis 1976, 259-261.

6. Nakamura, E.; Murotushi, T.; Shimizu, M.; Kuwajima, I. J. Am. Chem. Soc. 1976, 98, 2346-2348.

7. Chu, D. T.; W. Huckin, S. N. Can. J. Chem. 1980, 58, 138-142.

8. Torkelson, S.; Ainsworth, C. Synthesis 1976, 722-724.

9. Aizpurua, J. M.; Palomo, C. Synthesis 1982, 280-281.

10. Yamamoto, Y.; Matui, C. Organometallics 1997, 16, 2204-2206.

11. Rasmussen, J. K.; Heilmann, S. M.; Krepski, L. R. Advances in Silicon Chemistry, Larson, G.L., Editor. JAI Press INC. London, England, 1991, 1, 64-187.

12. (a) Furin, G. G.; Vyazankina, O.; A.; Gostevsky, B. A.; Vyazankin, N. S. Tetrahedron 1988, 44, 
2675-2749; (b) Colvin, E. W. Chem. Soc. Rev. 1978, 7, 15; (c) Groutas, W. C.; Felker, D. Synthesis 1980, 861-868.

13. (a) Mai, K.; Paril, G. J. Org. Chem. 1986, 51, 3545-3548; (b) Corey, E. J.; Wu, Y.-J. J. Am. Chem. Soc. 1993, 115, 8871-8872; (c) Becu, C.; Reyniers, M.-F.; Anteuuuunis, M. J. O. Bull. Soc. Chim. Belg. 1990, 99, 779-782; (d) Antenuis, M. J. O.; Becu, C.; Becu, F. Bull. Soc. Chim. Belg. 1990, 99, 361-377.

14. Zhuo, J.-C.; Wyler, H. Helv. Chim. Acta 1993, 76, 1916-1927.

15. (a) Gostevskii, B. A.; Kruglaya, O. A.; Albanov, A. I.; Vyazankin, N. S. Zh. Obshch. Khim. 1981, 51, 817-820; (b) Gostevskii, B. A.; Vyazankina, O. A.; Vyazankin, N. S. Zh. Obshch. Khim. 1983, $53,1843-1846$.

16. Foley, L. H. J. Org. Chem. 1985, 50, 5204-5209.

17. Neef, H.; Müller, R. J. Prakt. Chem. 1973, 315, 367-374

Samples Availability: Not available.

(C) 1999 by the authors. Reproduction of this article, by any means, is permitted for noncommercial purposes. 